\title{
Performance validation of phase diversity image reconstruction techniques
}

\author{
J. Hirzberger ${ }^{1}$, A. Feller ${ }^{1}$, T. L. Riethmüller ${ }^{1}$, A. Gandorfer ${ }^{1}$, and S. K. Solanki ${ }^{1,2}$ \\ 1 Max-Planck-Institut für Sonnensystemforschung, 37434 Katlenburg-Lindau, Germany \\ e-mail: hirzberger@mps .mpg.de \\ 2 School of Space Research, Kyung Hee University, Yongin, Gyeonggi 446-71, Korea \\ Received 8 September 2010 / Accepted 23 March 2011
}

\section{ABSTRACT}

\begin{abstract}
We present a performance study of a phase diversity (PD) image reconstruction algorithm based on artificial solar images obtained from MHD simulations and on seeing-free data obtained with the SuFI instrument on the SunRIse balloon borne observatory. The artificial data were altered by applying different levels of degradation with synthesised wavefront errors and noise. The PD algorithm was modified by changing the number of fitted polynomials, the shape of the pupil and the applied noise filter. The obtained reconstructions are evaluated by means of the resulting rms intensity contrast and by the conspicuousness of appearing artifacts. The results show that PD is a robust method which consistently recovers the initial unaffected image contents. The efficiency of the reconstruction is, however, strongly dependent on the number of used fitting polynomials and the noise level of the images. If the maximum number of fitted polynomials is higher than 21 , artifacts have to be accepted and for noise levels higher than $10^{-3}$ the commonly used noise filtering techniques are not able to avoid amplification of spurious structures.
\end{abstract}

Key words. Sun: photosphere - techniques: high angular resolution - methods: data analysis

\section{Introduction}

High-resolution solar observations are intimately connected with procedures measuring and reconstructing wavefront deformations produced by the Earth's atmosphere (seeing) and optical systems (telescopes and post-focus instrumentation). In principle, these procedures can be divided into real-time wavefront sensing and correction systems, such as adaptive optics and multi-conjugate adaptive optics (for a review and references see Glindemann et al. 2000) and post-facto reconstruction techniques. The latter methods are based on mathematical procedures (speckle interferometry, phase diversity wavefront sensing, blind deconvolution, etc.) that estimate and deconvolve the aberrations from the obtained scientific data (de Boer et al. 1992; Löfdahl \& Scharmer 1994; Schulz 1993; von der Lühe 1993).

Phase diversity (PD) techniques are based on deriving the wavefront deformations from single or bursts (speckle series) of images pairs of the same target with a certain known amount of defocus between the images (Gonsalves \& Chidlaw 1979). Within the fitting process, the wavefront is expanded in a series of polynomials adequately representing optical aberrations. Usually Zernike polynomials (see e.g. Noll 1976) or regularised Zernike polynomials (e.g. by means of Karhunen-Loève functions, see Roddier 1990) are used.

Although the power of PD reconstruction techniques has been confirmed by the recent spectacular findings in highresolution solar physics, mainly from observations obtained at the Swedish Solar Telescope (Scharmer et al. 2003) and from reconstructions with the frequently applied MOMFBD code (van Noort et al. 2005), this method, naturally, has several drawbacks. A major limitation is the contribution of noise which has to be treated adequately. A second limitation is the irregular wavefront deformation in seeing-limited observations. Both effects cause small-scale variations in the wavefront estimates which result in an ill-posed problem and do not lead to a unique solution when expanding into Zernike polynomials. These limitations tend to keep the number of fitted Zernike modes low. A usually applied technique to reduce the influence of these effects is the reconstruction of bursts (speckle series) of focusseddefocussed image pairs (Löfdahl et al. 1998).

Irrespective of the success of PD reconstruction techniques, careful performance studies, by means of "artificial" solar data are needed and have so far been carried out in a limited sense (Vargas Domínguez 2008). In the present paper a performance study of a PD code developed for reconstruction of data obtained with the Sunrise Filter Imager (SuFI, see Gandorfer et al. 2011) onboard the balloon-borne SunRISE solar telescope (Barthol et al. 2011; Solanki et al. 2010) is carried out. Basic parameters of the SuFI instrument are given in Table 1 . The focussed-defocussed image pairs are obtained by a PD image doubler based on two parallel and inclined glass plates mounted close to the science focus, so that the two images were obtained simultaneously, each on one half of the detector.

We apply theoretically synthesised wavefront deformations to artificial solar images obtained from magneto-hydrodynamic (MHD) simulations and describe the behaviour of the subsequently performed PD reconstruction. In addition, we show the performance of the thus optimised code when applied to real data obtained with the SunRISE/SuFI instrument.

\section{Wavefront synthesis and simulated observations}

To study the performance of PD reconstruction on artificial data the expected wavefront aberrations have to be simulated. Model simulations of the wavefront error budget of the SUNRISE observatory (except for the main mirror) were presented 


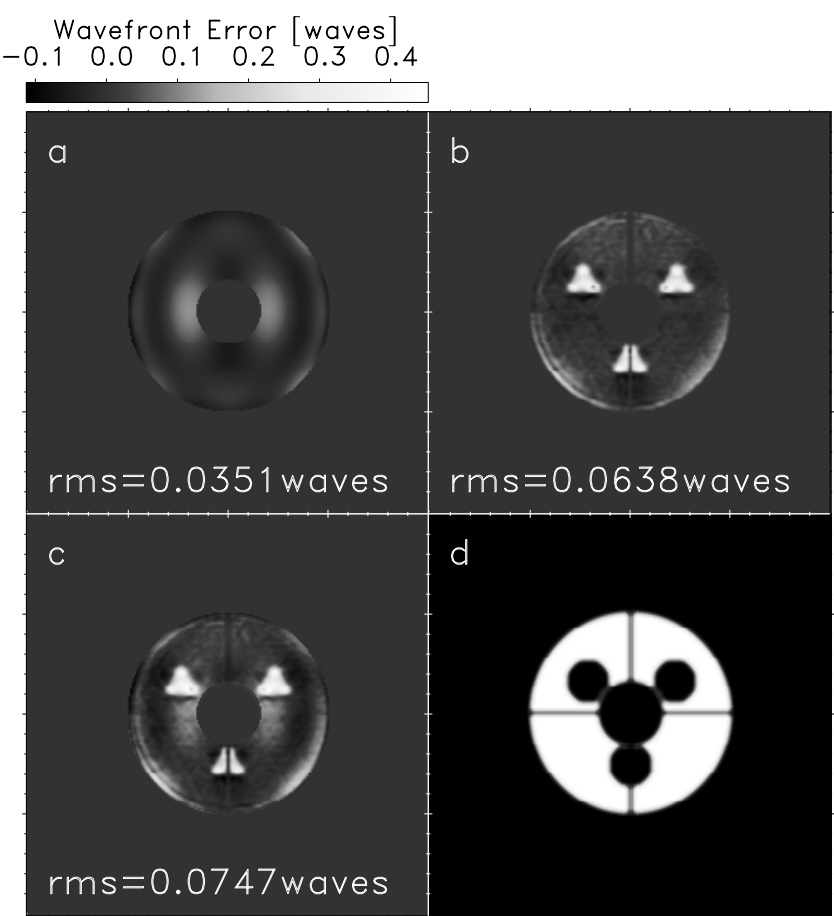

Fig. 1. Expected wavefront error budget (in waves at $388 \mathrm{~nm}$ ) for the SunRIse/SuFI instrument as obtained from model simulations. a) Telescope (excluding main mirror) and instrument; b) main mirror; c) telescope (including main mirror) and instrument optics; d) pupil mask applied in the PD algorithm.

Table 1. Basic parameters of the SunRISE/SuFI instrument.

\begin{tabular}{lr}
\hline \hline pupil diameter & $1 \mathrm{~m}$ \\
effective focal length & $121 \mathrm{~m}$ \\
PD defocus & $28.15 \mathrm{~mm}=1.05$ waves @ $214 \mathrm{~nm}$ \\
& $=0.58$ waves @ $388 \mathrm{~nm}$ \\
field of view & $20^{\prime \prime} \times 40^{\prime \prime}$ \\
plate scale & $\sim 00^{\prime \prime} 02 /$ pixel \\
spectral channels & $214,300,312,388,397 \mathrm{~nm}$ \\
\hline
\end{tabular}

by Vargas Domínguez (2008). In addition, measurements of the wavefront aberrations of the main mirror were carried out by Kampf (2008). In Fig. 1 an example of the total expected instrumental wavefront errors of the SUNRISE observatory is shown. The highest amplitude of wavefront errors is visible within three triangular structures produced by the support structure of the main mirror. The figure also shows the geometry of the secondary mirror assembly (leading to a roundish pupil obscuration of 0.332 times the pupil size) and the spider which supports the assembly.

Artificial solar observations were derived from numerical MHD simulations of the solar photosphere carried out with the MURaM code (Vögler et al. 2005). A simulation run with a mean vertical magnetic field strength of $\left\langle B_{z}\right\rangle=200 \mathrm{G}$ and a horizontal cell size of $20.8 \mathrm{~km}$ was used to produce intensity maps in the $388 \mathrm{~nm}$ band. These artificial filtergrams were obtained by performing non-grey radiative transport calculations for a full spectral synthesis with the SPINOR code (Solanki 1987; Frutiger et al. 2000; Berdyugina et al. 2003), including all available atomic and molecular line parameters within the $388 \mathrm{~nm}$ spectral bandpass of the SuNRISE/SuFI instrument.

Since the lateral boundary conditions of the MHD data are periodic, it was possible to make mosaics of several
MHD boxes. The thus obtained mosaics of artificial filtergrams were remapped (via cubic convolution interpolation) to the SuFI plate scale (0."02 which corresponds to $14.4 \mathrm{~km}$ on the Sun) and they were convolved with point spread functions (PSFs) obtained from the synthesised wavefronts. For testing the PD code, MHD image sizes of $512 \times 512$ pixels were used. Examples of such artificial images are shown in Fig. 2.

\section{The PD code}

The baseline PD code used in the present study was presented by Löfdahl \& Scharmer (1994) and further developed as described by Bonet et al. (2004), Criscuoli et al. (2005) and Vargas Domínguez (2008). This code has been originally developed for a round and unobstructed telescope aperture, i.e. it fits the wavefront aberrations by a set of Zernike polynomials which suffer loss of orthonormality when the pupil is obstructed as in a Gregorian configuration as e.g. the SunRISE telescope.

Although PD does not require an orthononormal basis of functions, in the algorithm used for the present study the obstructed Zernike polynomials were re-orthonormalised (applying singular value decomposition as described by Press et al. 2007). Hence, all the Zernike cefficients given in the present study (see Figs. 3 and 11 below) correspond to original Zernike polynomials (as defined, e.g., in Noll 1976), obtained after tranforming the coefficients from the re-orthonormalised basis of functions into the basis of original Zernike polynomials.

Irrespective of the pupil obscuration, the expected wavefront aberrations might contain localised peaks, such as the three triangular structures produced by the support of the main mirror in SunRISE (see Fig. 1b). Such localised peaks do not have a large contribution to the effects on the image quality since the fraction of the pupil area covered by these peaks is negligible (only about $4.5 \%$ ). This can be illustrated by a worst case scenario induced by the localised wavefront deformations: In the worst case all the light coming from the respective areas will strike the detector at the wrong position. This effect can be expressed by extended wings of the PSF, i.e. it is identical to increased straylight. Since the main mirror sees the entire solar disk the amount of additionally generated straylight can be, in principle, up to $4.5 \%$ of the light coming from the entire disk. In practise, the amount of additional straylight will be far below this value. Anyway, the localised wavefront deformations are poorly fitted by a limited number of polynomials. Each wavefront modelled by a limited set of orthonormal functions would tend to smear out these peaks so that a large fraction of the pupil area would be affected, resulting in strong deviations from the true wavefront and biasing the final image quality.

In order to circumvent the problems introduced by fitting the localised peaks in the wavefront, the pupil can be masked by excluding the affected regions from the fitting process. An example for such a masked pupil, including the central obscuration, the spider, and the positions of the main mirror supports is shown in Fig. 1d. After fitting the wavefront deformation, the images have to be reconstructed by using the pupil of the telescope, i.e. without masking the mirror supports. Neglecting the spider, the image reconstruction is carried out using an annular pupil.

\section{Performance of the PD code}

A critical part in a PD code is the used noise filter. Usually, noise filters are calculated from the power spectra of the input data (see e.g. Löfdahl \& Scharmer 1994). In order to avoid effects from 


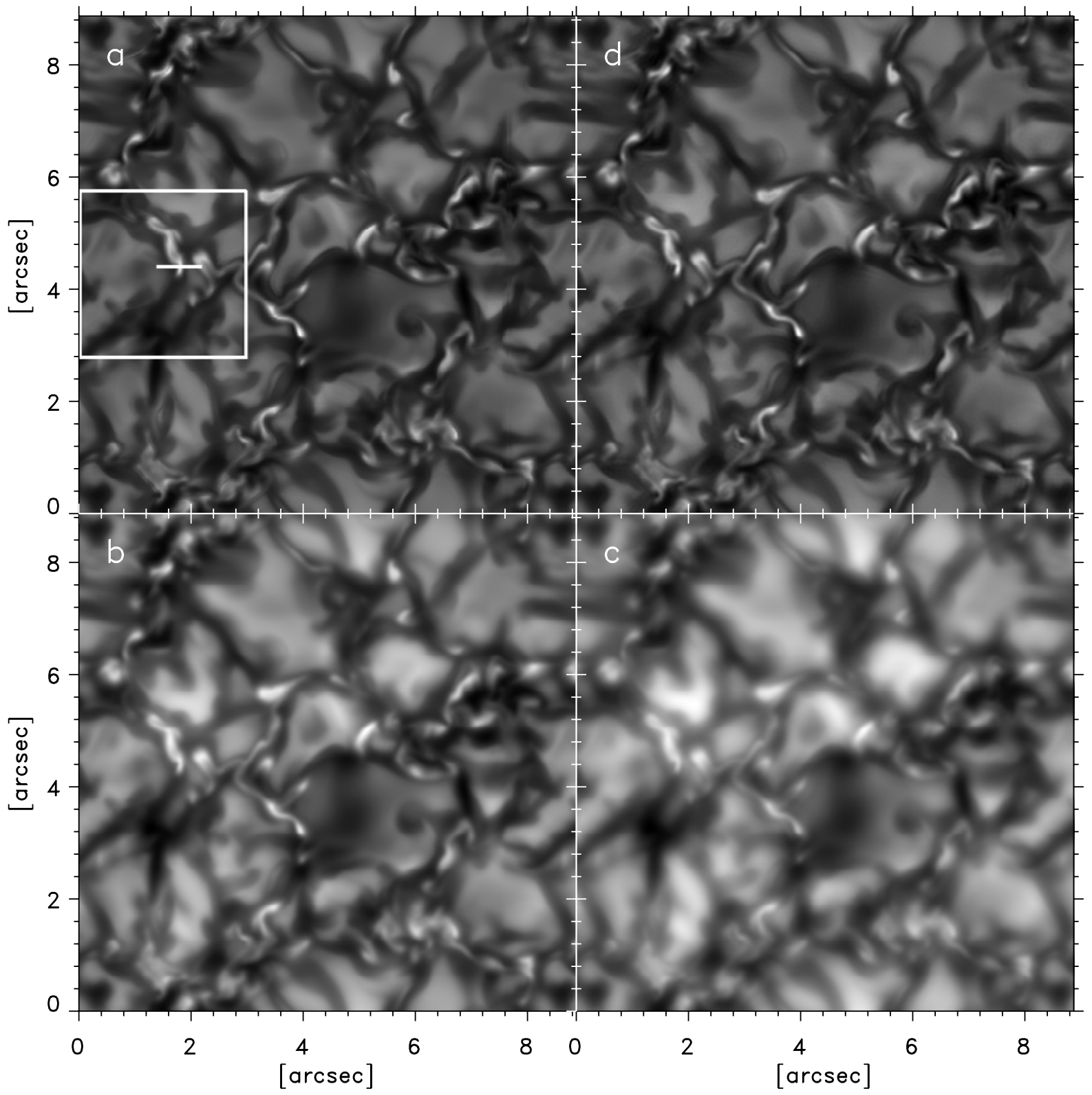

Fig. 2. a) Artificial solar image in the $388 \mathrm{~nm}$ band as obtained from MHD simulations and non-grey radiative transport calculations after remapping to the SuFI plate scale; b) MHD image degraded by convolving with the wavefront errors displayed in Fig. 1c and by adding a noise level of $10^{-3}$; c) MHD image degraded with an additional defocus of 0.58 waves; d) PD reconstruction of panels b) and c) for $N=21$ and applying a masked pupil. The bold white line around position $\left(1{ }^{\prime \prime} 8,44^{\prime \prime} 2\right)$ in panel a) denotes a cut through a bright point discussed in Sect. 4.1 and Fig. 6 . The white square marks the detail shown in Fig. 8.

different noise filters we therefore used for all reconstructions of MHD data presented in Sect. 4.1 a circular filter with a sharp cutoff at $0 .{ }^{\prime \prime} 07$, instead of re-calculating the filter in each iteration (cf. Eq. (18) in Löfdahl \& Scharmer 1994). The cut-off was set to be slightly below the diffraction limit of an $1 \mathrm{~m}$ aperture at $388 \mathrm{~nm}$.

\subsection{Annular vs. masked pupil}

The performance of the PD code was tested on the basis of the numerical MHD simulations described in Sect. 2. Figures 3-5 show the performance when applying an annular and a masked pupil, respectively. As an idealised case, the corresponding input data were produced by degrading the MHD image with the measured wavefront errors of the SUNRISE main mirror only and without adding noise. The thus obtained input data
(10 MHD snapshots) were reconstructed by dividing each of them into nine overlapping sub-boxes of $5^{\prime \prime} \times 5^{\prime \prime}$ ( 256 pixels $\times 256$ pixels $)$ size. This was done in order to obtain a large number of statistically independent reconstructions. Moreover, the required CPU times can be decreased significantly when dividing the images into overlapping sub-boxes.

Applying an annular pupil and using $N=21$ polynomials, the resulting wavefront errors averaged over all of the 90 subboxes from the 10 MHD snapshots show two main contributions: Zernike term $n=9$ ( $y$-trefoil) and Zernike term $n=11$ (spherical aberration). The wavefront errors obtained by using the masked pupil also show two main components: Zernike terms $n=7$ (y-coma) and $n=9$ (y-trefoil). The scatter is slightly smaller using the masked pupil than with the annular one although the standard deviations for terms $n \gtrsim 16$ are high for both methods. This is a sign for the ambiguity appearing when 

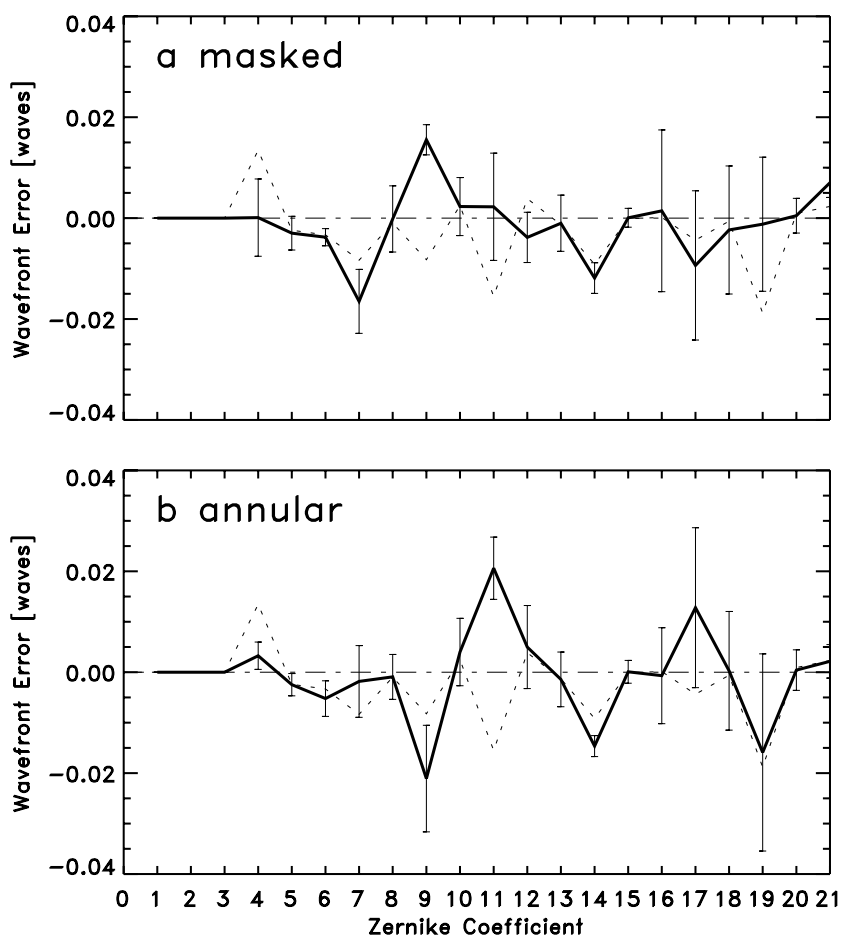

Fig. 3. Reconstructed wavefront errors of 10 MHD images degraded with the wavefront errors produced by the main mirror of SUNRISE only. The figure shows the coefficients of original Zernike polynomials averaged over 10 MHD snapshots, each subdivided into nine sub-boxes, and the corresponding standard deviations. The dotted curves show a decomposition of the true wavefront into Zernike polynomials.

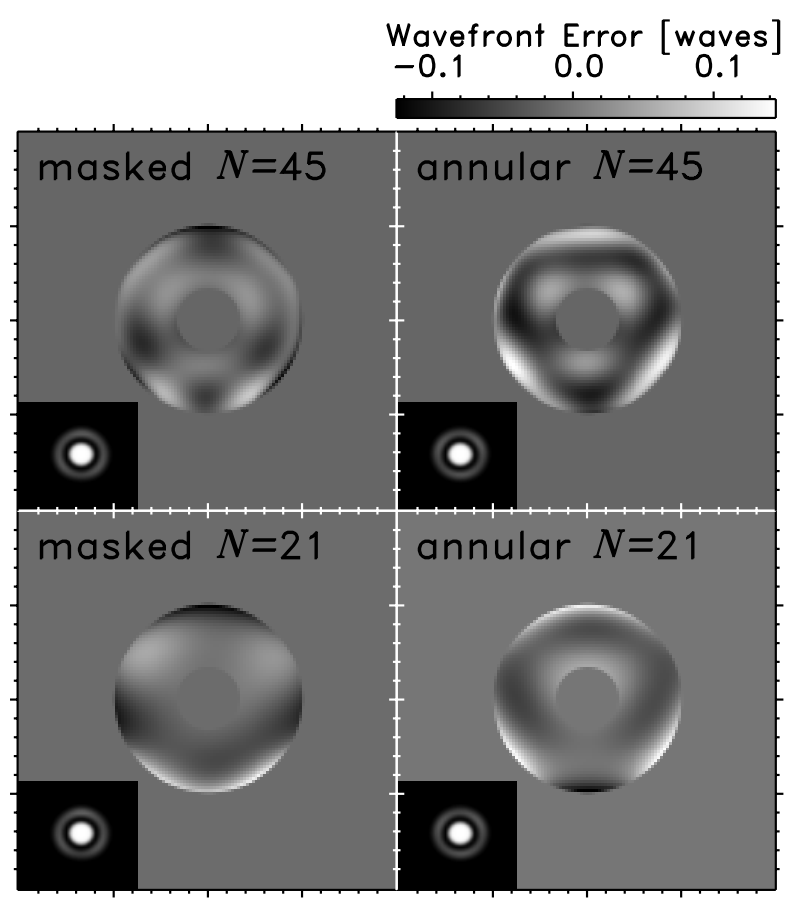

Fig. 4. Mean wavefront errors on the pupil obtained from reconstruction of 10 MHD images degraded with the wavefront errors produced by the main mirror of SunRISE (cf. Figs. $1 \mathrm{~b}$ and 3). The inserts in the lower left corners represent the point spread functions obtained from the wavefront errors. The inserts are 0.7 in size.

reconstructing the true wavefront errors with an ill-posed set of polynomials. As can be seen from a comparison with a Zernike decomposition of the true wavefront (dotted lines in Fig. 3),
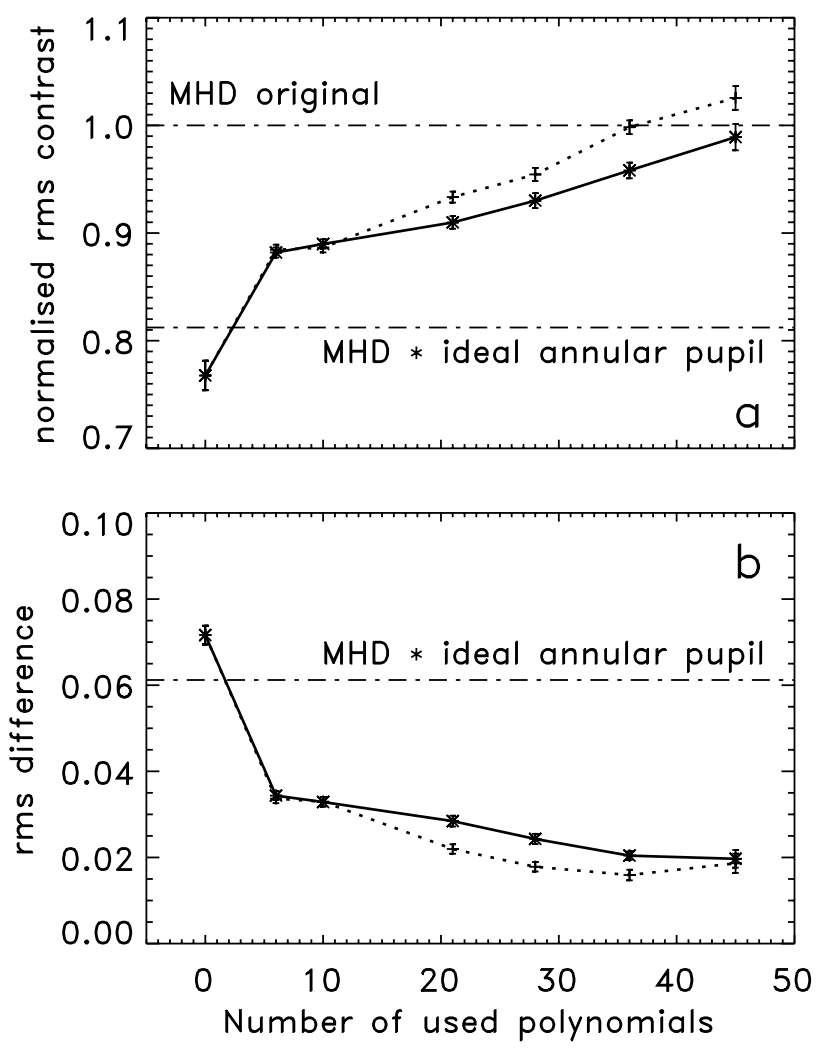

Fig. 5. a) Mean rms intensity contrasts, obtained from MHD images which are artificially degraded with the wavefront errors produced by the main mirror of SunRISE (cf. Fig. 1b) and subsequently PD reconstructed. rms values are normalised to that of the original MHD images. Error bars denote the corresponding standard deviations obtained from applying the procedure to 10 different snapshots. The solid curves were obtained using a masked pupil and the dotted curves stem from using an annular pupil. The contrast of the images corrected only for diffraction at the entrance pupil is given by the leftmost data point (zero fit coefficients). b) Mean contrasts of the difference images $\left(I_{\mathrm{MHD}}-I_{\mathrm{PD}}\right) / I_{\mathrm{MHD}}$. The dash-dotted curves show the normalised contrast of the original MHD image and the contrast after convolution with the PSF of an ideal annular telescope, averaged over the 10 MHD snapshots.

the obtained Zernike coefficients show significant deviations. However, it was already mentioned by Löfdahl \& Scharmer (1994) that a PD reconstruction produces better results with a wavefront where with wrong low-order terms high-order contributions are simulated than with a truncated set of coefficients obtained from a decomposition of the true wavefront.

In Fig. 4 the averaged fitted wavefront error distributions on the pupil are displayed. Comparison with Fig. 1b shows clearly that it is impossible to model the mirror support structures with only 21 Zernike polynomials. Increasing the number of polynomials to $N=45$, the mirror support structures become partly recognisable in the fitted wavefront errors. Particularly, when the annular pupil is used, the fitted wavefront bears visual resemblance to the true wavefront errors displayed in Fig. 1b. However, although the visual impression shows an advantage of the annular pupil with respect to the masked pupil, the quantitative deviations to the true wavefront are only marginally smaller (rms values amount to $\delta\left(W F E_{\text {true }}-W F E_{\text {ann }}\right)_{\mathrm{rms}}=5.66 \times$ $10^{-2}$ waves and $\delta\left(W F E_{\text {true }}-W F E_{\text {mask }}\right)_{\text {rms }}=6.30 \times 10^{-2}$ waves, respectively). Again the obtained errors are rather high since with the low number of polynomials only large-scale trends of the wavefront can be reproduced. The small-scale wavefront 

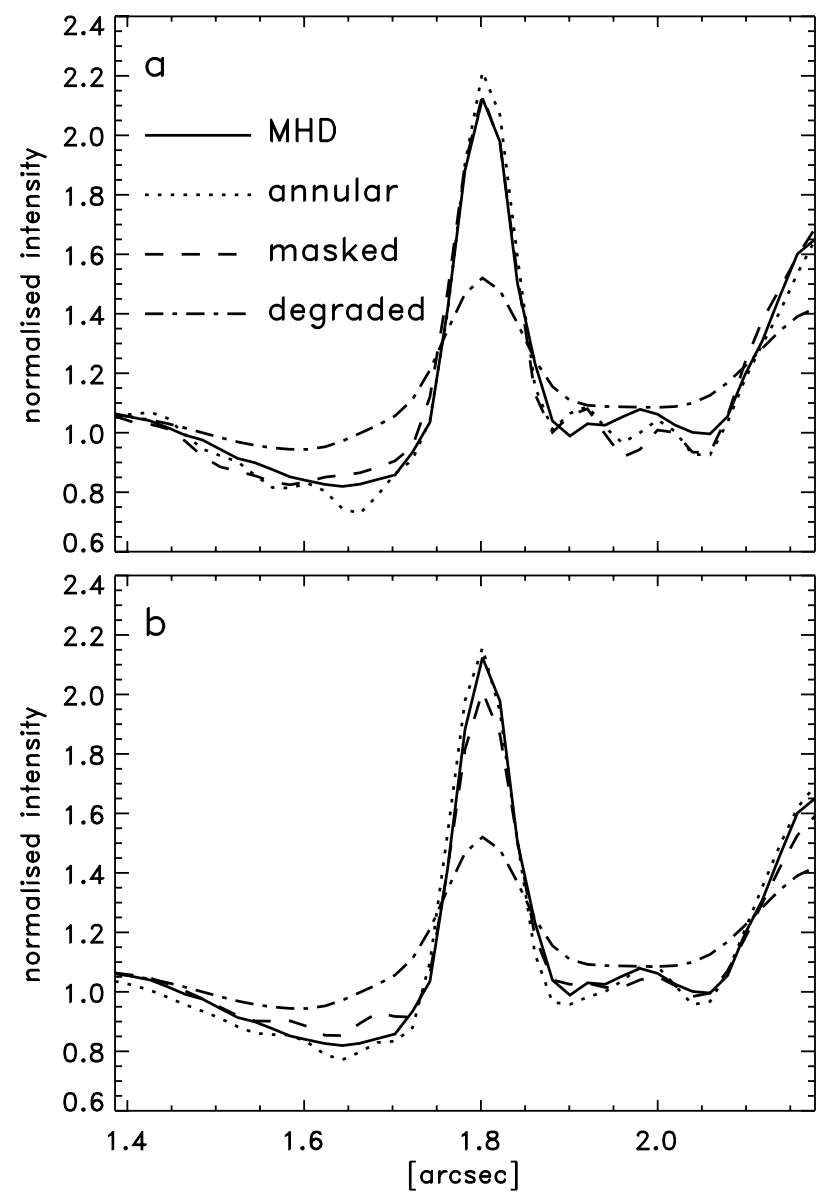

Fig. 6. Normalised intensity of the bright point marked by a white line in Fig. 2, taken from the original MHD data (solid lines) and after degrading the image with the wavefront errors as shown in Fig. 1b (dashdotted lines). Panels a) and b) show reconstructions with $N=45$ polynomials and $N=21$ polynomials, respectively.

deformations are responsible for the large error values in the above stated differences but they hardly affect the spatial resolution (as can be seen in Fig. 2d). As mentioned above, small-scale wavefront deformations produce straylight which reduces the image contrast but not the overall spatial resolution. The point spread functions (PSFs) obtained from the fitted wavefront errors are shown in the inserts of Fig. 4. It can be seen that their cores are practically identical. Their shapes are dominated by the diffraction at the annular pupil with a central peak of 0 ." 1 diameter (diffraction limit of an 1-m pupil) and an annular structure of approximately 0.'29 width (diffraction at the central obscuration). Only in the far wings slight differences can be detected. This means that the resulting PD reconstructions are rather similar with slight differences in the obtained rms contrasts since the wings of the point spread functions can be interpreted as straylight contributions produced by small-scale deformations of the wavefront which cannot be fully reproduced with a limited set of polynomials.

Figure 5a shows the performance of the PD code by means of the rms intensity contrast of the reconstructed images $\delta I_{\mathrm{PD}, \mathrm{rms}}$, normalised to the rms contrast of the original MHD images. Figure $5 \mathrm{~b}$ shows the rms fluctuations of the normalised differences between original MHD images and reconstructions, $\delta\left(\left(I_{\mathrm{MHD}}-I_{\mathrm{PD}}\right) / I_{\mathrm{MHD}}\right)_{\mathrm{rms}}$. The used numbers of polynomial coefficients $(N=0,6,10,21,28,36$, and 45 from which only the numbers $[4 \leq n \leq N]$ are fitted) represent azimuthal symmetries for radial orders $m=2 \ldots 8$ for the original Zernike basis (see e.g. Noll 1976). The rms contrast of the original MHD images is between $23.3 \%$ and $25.7 \%$. In Fig. 5 a all rms contrasts were normalised to these original values. Irrespective of the number of the fitted coefficients, the resulting contrasts lie always above those of images resulting from convolutions with the point spread function of an ideal annular pupil (between $18.67 \%$ and $21.14 \%)$. The figure shows clearly that the shape of the pupil has no influence when using only a small number (e.g. 6 or 10) of coefficients. For a larger number of coefficients, the contrast of the reconstructed image is higher for an annular pupil than for the masked one. This excess in contrast even leads to a systematic overshoot of the original MHD contrast when using 45 polynomials, thus showing a strong tendency of the method to over-reconstruct the image and to produce artificial structures if the annular pupil is used. The rms differences between original and reconstructed images (Fig. 5b) are continuously decreasing with increasing $N$ until $N=36$ and the reconstructions using an annular pupil show less deviations from the original MHD images. For a larger number of polynomials the advantage of the annular pupil disappears and the corresponding differences are increasing. This is another hint for the higher susceptibility to produce artifacts due to over-reconstructions when using an annular pupil.

Over-reconstruction is illustrated in Fig. 6 by the intensity of a bright point. It can be seen that the reconstruction with an annular pupil leads to bright point intensities higher than in the original MHD data both for $N=21$ and $N=45$. The dark lanes beside the bright point are too faint in these reconstructions. For $N=45$, both pupils produce intensity oscillations nearby the bright point which are not visible in the original data. These oscillations are less pronounced when using the masked pupil. This pupil also does not lead to an overly large rms contrast or an over-reconstruction of the bright point.

\subsection{Application to non-ideal data}

Simulating more realistic solar observations requires including noise and accounting for more contributions to the wavefront errors. The MHD snapshots were, therefore, degraded with the wavefront errors shown in Fig. 1c, i.e. the effects from the entire telescope/instrument optics, and different noise levels were added. Averaged rms contrasts, normalised to the original values, obtained from reconstructing 10 MHD snapshots with both masked and annular pupils, vs. the number of the highest order Zernike polynomial, $N$, are shown in Figs. 7a-c. Surprisingly, the resulting rms contrasts depend only weakly on the noise level, which is a strong indication for the robustness of the used method. Nevertheless, the obtained contrasts are systematically higher for the more noisy data, implying a considerable amplification of noise, in particular if the noise level is set to $\delta \xi_{\text {rms }}=10^{-2}$ and the number of fitted polynomials is high. The added noise is assumed to be normally distributed with a standard deviation of $\delta \xi_{\text {rms }}$ with respect to the mean intensity of the artificial data. In Figs. 7d-f the rms differences between original MHD images and reconstructions are plotted. The results are rather similar to those using ideal data, as shown in Fig. 5b, except that the absolute values are somewhat higher and increase with the noise level. The general trend of reduced differences with increasing number of fitted polynomials becomes reversed for a noise level of $10^{-2}$ : for a very large number of polynomials $(N>21)$ the differences between original and reconstructed images starts to increase again. 

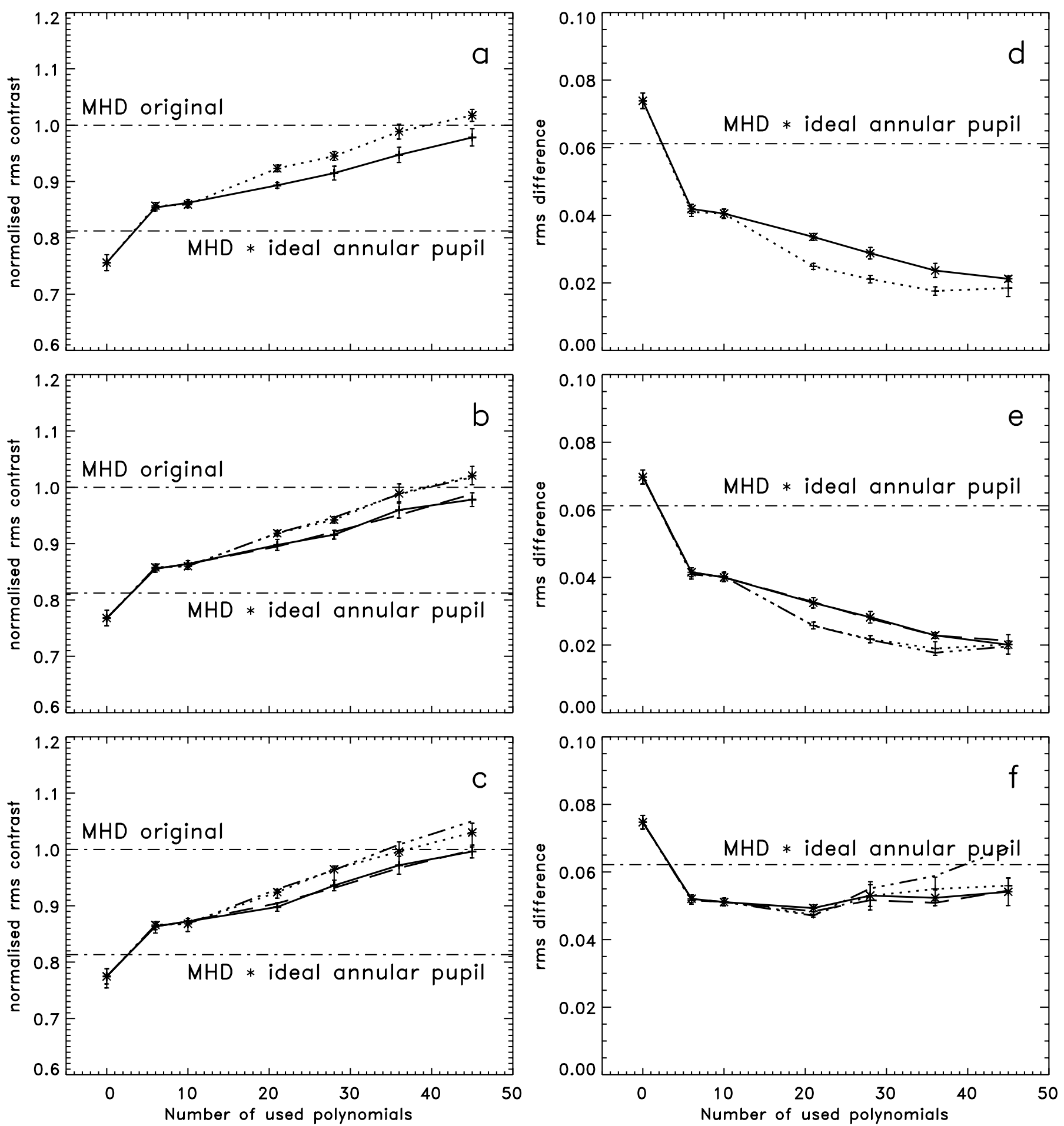

Fig. 7. Averaged normalised rms contrasts of PD reconstructions of 10 MHD snapshots degraded with the wavefront errors shown in Fig. 1c. The noise levels were set to zero (panel a)), $10^{-3}$ (panel b)) and $10^{-2}$ (panel c)). Solid lines correspond to masked-pupil reconstructions, dotted lines denote annular-pupil reconstructions. The dashed and the dash-dot-dot-dotted line in panels (b)) and (c)) were obtained from applying an optimum noise filter instead of a fixed cut-off and masked and annular pupils, respectively. d)-f) Same as a)-c) but for mean contrasts of the difference images $\left(I_{\mathrm{MHD}}-I_{\mathrm{PD}}\right) / I_{\mathrm{MHD}}$. The dash-dotted curves show the normalised contrast of the original MHD image and the contrast after convolution with the PSF of an ideal annular telescope, averaged over the 10 MHD snapshots.

The amplification of the noise is demonstrated in the difference images (reconstructed minus original MHD image) shown in Fig. 8. The displayed details show clearly that the applied noise filtering is not sufficient when the noise levels is $10^{-2}$. The effect of noise amplification appears already when using only $N=6$ polynomials in the reconstruction process and becomes worse with increasing $N$. For $N=45$ the difference images are fully dominated by noise structures. When the noise level is lower $\left(\delta \xi_{\mathrm{rms}}=10^{-3}\right)$, the amplification of noise is much weaker and starts to become significant in the difference images only for $N \geq 28$. Interestingly, the effect is much more serious when using the annular pupil instead of the masked one. 
J. Hirzberger et al.: Performance validation of phase diversity image reconstruction techniques

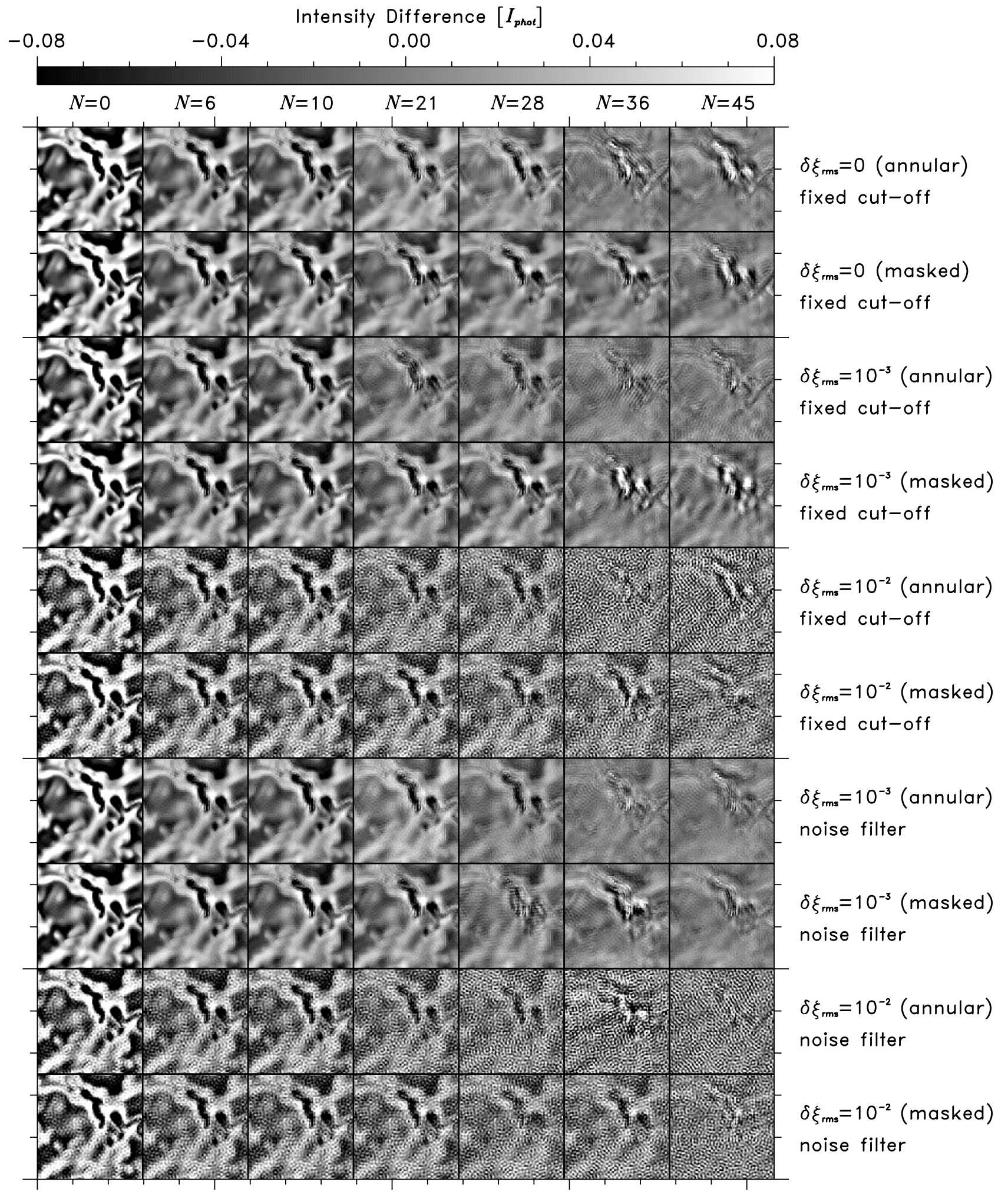

Fig. 8. Intensity difference of details (white square in Fig. 2) of the original and the reconstructed MHD images applying different noise levels and numbers of fitted polynomials. Each image was normalised to its mean intensity, $I_{\text {phot }}$, before building the difference. Tickmarks are given in arcseconds.

From Fig. 7 it might be guessed that these effects could be reduced by using an optimum noise filter, re-calculated at each iteration (see Löfdahl \& Scharmer 1994), instead of using a fixed cut-off. As can be seen clearly in Figs. 7a-c, the different shapes of the noise filters produce almost similar rms contrasts. This becomes also obvious when looking at the difference images displayed in Fig. 8. The contamination of noise in the reconstructed images is similar to that when applying the fixed cut-off.

If applied to real solar observations, this effect might be even more pronounced since observational data usually contain more disturbances than pure white noise. Another effect clearly visible in Fig. 8 is that in the difference images for 


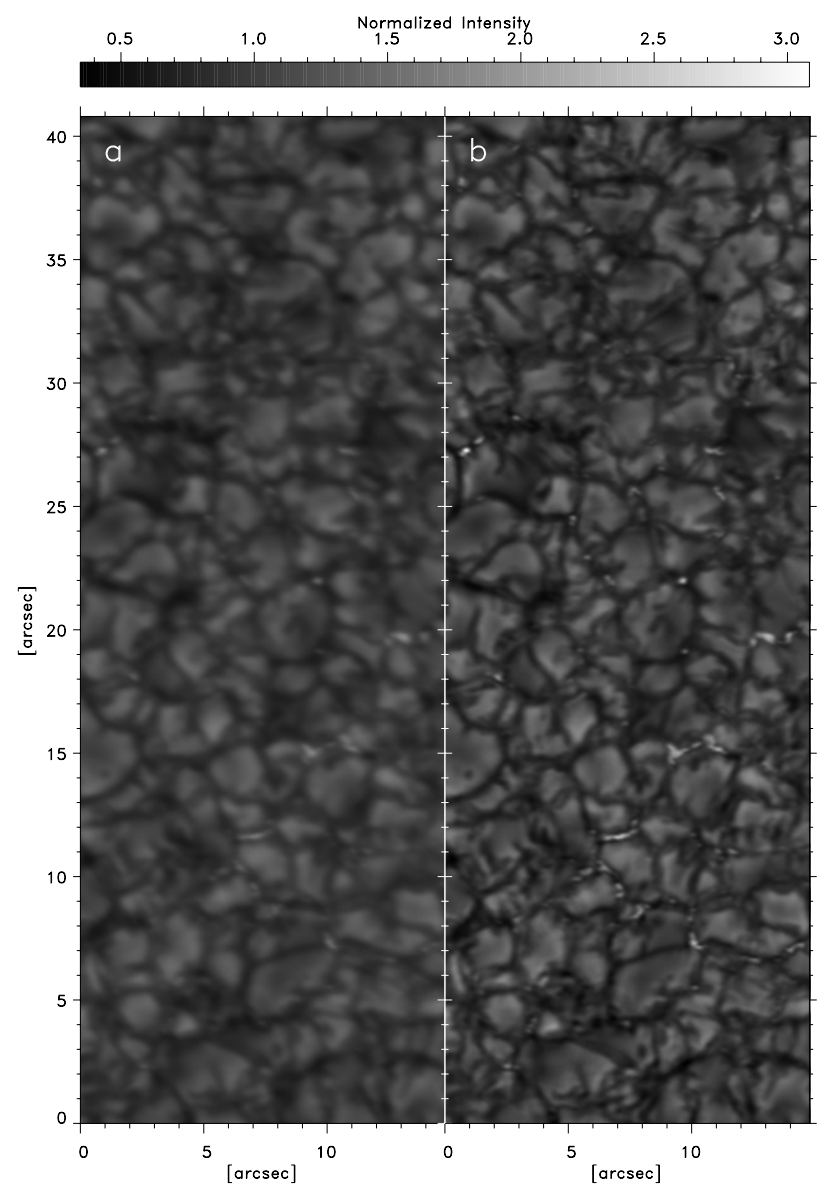

Fig. 9. Example PD reconstruction of real solar data: a) unreconstructed SunRISE/SuFI image obtained on June 11, 2009 in the SuFI-300 nm band; b) PD reconstruction using a masked pupil and $N=21$. Both images are normalised to their mean intensities and scaled to the same grey levels.

$N=45$ appears a periodic intensity pattern which is not visible for smaller $N$. This periodic pattern is clearly an artifact which means that even when the images are not dominated by noise, over-reconstructions become an issue when using a high number of fitted polynomials.

From the results presented in this section, it might be concluded that with $N=21$ the maximum achievable restoration is not reached, but the danger of producing artificial structures is also low. For low noise levels the shape of the noise filter has a minor influence whereas efficient noise filtering becomes essential for data suffering from a high noise level. Therefore, using $N=21$ represents a conservative choice that still gives good results. In Fig. $2 \mathrm{~d}$ a PD reconstructed image after adding a noise level of $10^{-3}$ and applying a masked pupil and $N=21$ is displayed. A noise filter with a cut-off at 0."07 was used. From pure visual inspection the differences to the original MHD image can hardly be identified although the rms contrast is $2.3 \%$ lower than for the original image.

\section{Application to observational data}

After demonstrating the robustness and the high efficiency of the PD method and the code presented here, we have applied the code to real solar observations. In Fig. 9 a quiet-Sun disk centre image obtained on June 11, 2009, in the $300 \mathrm{~nm}$ band of the Sunrise/SuFI instrument is shown. The left panel of Fig. 9
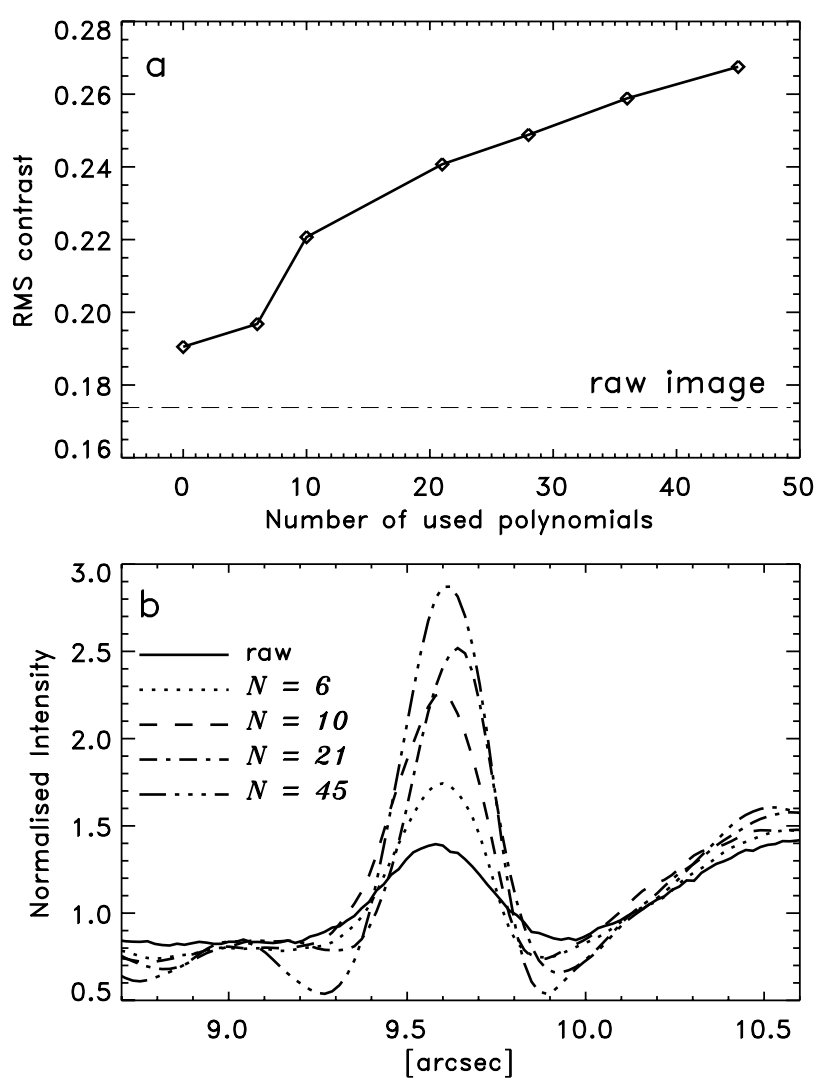

Fig. 10. a) rms contrast of $P D$ reconstructions of the image shown in Fig. 9 a vs. $N . N=0$ denotes correction only for diffraction at the entrance pupil. The dash-dotted horizontal line shows the rms contrast of the raw image. b) Horizontal cut through the bright point located at $\left(9{ }^{\prime \prime} 6,22.0\right)$ in Fig. $9 b$ in the raw image and in PD reconstructions with different $N$.

shows the raw image, corrected only for dark current and flat field. In the right panel of Fig. 9 a PD reconstruction applying the masked pupil and using $N=21$ is shown. The improvement of the overall image quality can be easily identified.

The variation of the rms contrast vs. the number of polynomials used for PD reconstruction of the image is plotted in Fig. 10a. The contrast rises steeply until $N=10$, flattens between $N=10$ and 21, before it again steeply increases to $N=28$. For higher $N$ the contrast increases only weakly. The same behaviour can be seen for the detail shown in Fig. 10b. The intensity of the bright point rises strongly until $N=10$ and shows only a weak increase between $N=10$ and 21. For $N=45$ the intensity is again much higher, in agreement with the reconstructions of images obtained from MHD simulations. For this large number of polynomials the code is either strongly over-reconstructing or it is able to fit small-scale features in the wavefront error distribution. According to the study presented in Sect. 4.1, over-reconstruction cannot be excluded. Therefore, we consider $N=21$ as the optimum number of polynomials obtained from a trade-off between contrast improvement and conservatively avoiding the occurrence of artificial structures in the resulting images.

\subsection{Performance stability tests on real data}

The data obtained from the first science flight of SUNRISE are an ideal case for studying the stability of PD reconstruction techniques on real data. At balloon altitudes the contributions from 
J. Hirzberger et al.: Performance validation of phase diversity image reconstruction techniques
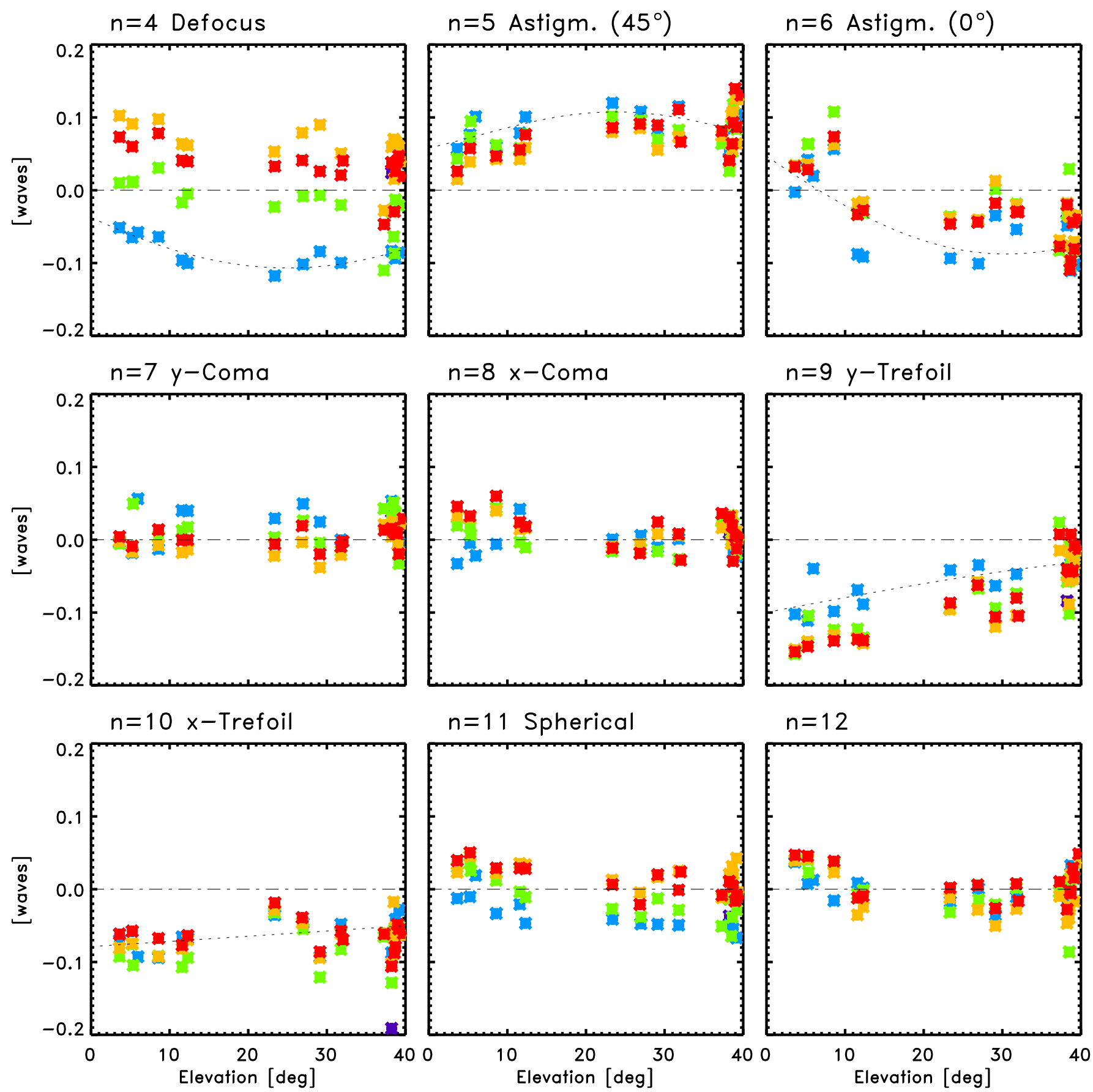

Fig. 11. Mean Zernike coefficients vs. solar elevation obtained from PD reconstructions of the SunRISE/SuFI data. The different colours correspond to the SuFI spectral bands: $300 \mathrm{~nm}$ (blue); $313 \mathrm{~nm}$ (green); $388 \mathrm{~nm}$ (yellow); $397 \mathrm{~nm}$ (red). The dotted curves denote sinusoidal fits to the $300 \mathrm{~nm}$ data.

residual seeing to the total wavefront errors is small compared to instrumental effects. Therefore, we expect isoplanatic conditions and temporal variations of the wavefront deformations arising only from changes of the instrument's temperature and from slowly varying mechanical deformations, which both are mainly correlated with the solar elevation.

In Fig. 11 the variations of 9 Zernike coefficients vs. solar elevation as obtained from SunRISE/SuFI data are plotted. Each data point denotes an average of the respective Zernike coefficients over all sub-boxes of all the useful images within one hour of observation. Only data obtained during periods of stable pointing of the observatory are considered. The figure shows clear trends of the wavefront errors with solar elevation. Since the observatory is not fully achromatic, offsets between the different spectral channels are expected. The chromatism can be best seen in the defocus component $(n=4)$. Although a slight trend with elevation can be recognised, the variation with solar elevation is small compared to the variation of the actual focal lengths of the different spectral channels.

The most prominent contributions to the obtained wavefront errors are astigmatism ( $n=5$ and 6) and trefoil ( $n=9$ and 10). Astigmatism shows extrema at medium elevations and weaker contributions at minimum and maximum elevation. The trefoils are always negative and tend to show linear trends with elevation. Particularly, the $y$-trefoil $(n=9)$ shows also a clear dependence on wavelength. This can be explained by the fact that 
the main mirror of the SUNRISE telescope shows geometrical deformations caused by the mirror supports (see Fig. 1b) which translate into wavelength dependant phase shifts. All other contributions are small and clear trends are not visible, except chromatism in the spherical term $n=11$. The origin of the different contribution to the averaged wavefronts can be seen best when rescaling the plots in Fig. 11 from units [waves] to [nanometres] (not shown). Then the chromatism for e.g. $n=9$ disappears almost completely but that for $n=4$ and $n=11$ is only slightly reduced. This indicates that the defocus is mainly due to chromatism of the refractive optical components (field lenses etc.), whereas trefoil is a geometrical effect, i.e. it might be indeed caused by the main mirror.

\section{Discussion and conclusions}

The present performance test of PD image reconstruction is carried out by degrading artificial solar data with optical aberrations as assumed to appear in the balloon borne SUNRISE solar observatory. The reconstructed data show a good correspondence with the original non-degraded MHD data although, for the first glance, the the fitted wavefront errors deviate considerably from the true wavefront errors. This is due to the effect that the highorder terms of the true wavefront errors cannot be fitted uniquely in a PD wavefront approximation algorithm. These terms have to be approximated by contributions to the low-order terms.

From an application to real solar data (cf. Fig. 11) it can be concluded that the obtained low-order approximations to the wavefront errors include those aberrations which produce the strongest image degradations. In addition, these wavefront errors are expected to appear from model simulations, although the obtained variations with elevation were not fully predictable. The trends obtained in Fig. 11 show that PD and, particularly, in the form implemented in the used code is a robust method for a dependable, although not perfect, approximation to the wavefront errors in solar imaging data. Tests involving reconstruction of simulated data also give rather robust results. Nevertheless, PD is usually based on an ill-posed problem and a certain scatter in the polynomial coefficients has to be expected and, consequently, the obtained rms contrasts depend on the number of fitted polynomials and the introduced noise level. However, the variation of the contrasts of a series of snapshots is small.

From the results based on simulated data, the optimum set of input parameters to the PD code can be retrieved. We have found some evidence that the optimum number of fitting polynomials depends somewhat on the noise level of the data. If both the noise level and the number of polynomials is high, then a strong amplification of noise has to be accepted. For noise levels smaller or equal $10^{-3}$ and $N=21$ no over-reconstruction effects have been detected. Using a larger number of polynomials may lead to intensities and contrasts higher than the original non-degraded MHD data in the presence of noise levels higher than $10^{-3}$.

Choosing this rather conservative approach, which tries to avoid any sign of over-reconstruction and artifacts in the reconstructed data results in an undeniable discrepancy between
PD reconstructed observations and MHD simulations. However, it has been shown by Hirzberger et al. (2010) that the theoretical rms contrast, obtained from quiet-Sun MHD simulations, in the $300 \mathrm{~nm}$ spectral band is around $30 \%$ and that the used data are affected from scattered light which may reduce the contrast down to about $25.5 \%$. This is only slightly above the contrasts of $24.2 \%$ obtained from PD reconstructed observations taking this conservative approach. A more detailed analysis of straylight contamination in SuFI/SunRISE data will be given by Feller et al. (in prep.).

In summary, PD techniques allow the reconstruction of solar imaging data such that they end up close to the unaffected solar scene. However, fine tuning of the PD parameters is essential in order to inhibit the appearance of artifacts. Note, that the tests and computations presented here have all been carried out in the absence of seeing, so that further investigations are required to judge to what extent the present results are applicable to groundbased data.

Acknowledgements. The authors are grateful to M. G. Löfdahl for valuable comments on the manuscript. The German contribution to SunRISE is funded by the Bundesministerium für Wirtschaft und Technologie through Deutsches Zentrum für Luft- und Raumfahrt e.V. (DLR), grant number 50 OU 0401, and by the Innovationsfond of the President of the Max Planck Society (MPG). This work has been partially supported by the WCU grant (No. R31-10016) funded by the Korean Ministry of Education, Science and Technology.

\section{References}

Barthol, P., Gandorfer, A., Solanki, S. K., et al. 2011, Sol. Phys., 268, 1 Berdyugina, S. V., Solanki, S. K., \& Frutiger, C. 2003, A\&A, 412, 513

Bonet, J. A., Márquez, I., Muller, R., Sobotka, M., \& Tritschler, A. 2004, A\&A, 423, 737

Criscuoli, S., del Moro, D., Bonet, J. A., \& Márquez, I. 2005, Sol. Phys., 228, 177

de Boer, C. R., Kneer, F., \& Nesis, A. 1992, A\&A, 257, L4

Frutiger, C., Solanki, S. K., Fligge, M., \& Bruls, J. H. M. J. 2000, A\&A, 358, 1109

Gandorfer, A., Grauf, B., Barthol, P., et al. 2011, Sol. Phys., 268, 35

Glindemann, A., Hippler, S., Berkefeld, T., \& Hackenberg, W. 2000, Exp. Astron., 10, 5

Gonsalves, R. A., \& Chidlaw, R. 1979, in Applications of Digital Image Processing III, ed. A. G. Tescher, SPIE Conf. Ser., 207, 32

Hirzberger, J., Feller, A., Riethmüller, T. L., et al. 2010, ApJ, 723, L154

Kampf, D. 2008, SUNRISE MT Full Aperture Interferometric Test, Tech. Rep., Kayser-Threde $\mathrm{GmbH}$, Munich

Löfdahl, M. G., \& Scharmer, G. B. 1994, A\&AS, 107, 243

Löfdahl, M. G., Berger, T. E., Shine, R. S., \& Title, A. M. 1998, ApJ, 495, 965

Noll, R. J. 1976, J. Opt. Soc. Am. (1917-1983), 66, 207

Press, W. H., Teukolsky, S. A., Vetterling, W. T., \& Flannery, B. P. 2007,

Numerical Recipes, 3rd edn. (Cambridge University Press)

Roddier, N. 1990, Opt. Engin., 29, 1174

Scharmer, G. B., Bjelksjo, K., Korhonen, T. K., Lindberg, B., \& Petterson, B. 2003, in SPIE Conf. Ser. 4853, ed. S. L. Keil, \& S. V. Avakyan, 341

Schulz, T. J. 1993, J. Opt. Soc. Am. A, 10, 1064

Solanki, S. K. 1987, Ph.D. Thesis, No. 8309, ETH, Zürich

Solanki, S. K., Barthol, P., Danilovic, S., et al. 2010, ApJ, 723, L127

van Noort, M., Rouppe van der Voort, L., \& Löfdahl, M. G. 2005, Sol. Phys., 228, 191

Vargas Domínguez, S. 2008, Ph.D. Thesis, Instituto de Astrofísica de Canarias, La Laguna

Vögler, A., Shelyag, S., Schüssler, M., et al. 2005, A\&A, 429, 335

von der Lühe, O. 1993, A\&A, 268, 374 